\title{
Pobreza material y antropológica: una aproximación desde la Doctrina Social de la Iglesia
}

\section{Material and Anthropological Poverty: an Approach from the Church's Social Doctrine}

\section{CRISTIAN MENDOZA \\ (Pontificia Universidad de la Santa Cruz, Roma) \\ cmendoza@pusc.it}

Resumen: Este artículo recoge en síntesis algunas de las principales perspectivas desde las cuales se comprende la pobreza. Se exponen las iniciativas de las Naciones Unidas y del Foro Económico Mundial, así como de algunos profesores y centros de investigación dedicados a este tema. El objetivo de este artículo es hacer una aproximación a este problema desde la Doctrina Social de la Iglesia para subrayar que se trata de un problema antropológico que va más allá de la escasez de recursos económicos. La pobreza puede traducirse en términos de violencia y corrupción, desprecio de la vida y de los más necesitados, etc., si se considera como un problema moral o antropológico. Esta pobreza antropológica da lugar a una pobreza económica, mientras que la pobreza económica no necesariamente genera pobreza antropológica o moral Además, en este documento se analizan algunas de las soluciones que se dan a la pobreza, subrayando la importancia de multiplicar las relaciones humanas en beneficio de un mayor saber hacer social. El desarrollo social se alcanza no cuando todas las personas hacen lo mismo, sino cuando cada persona sabe llevar a cabo de manera excelente su propia tarea.

Palabras clave: Pobreza, Doctrina Social de la Iglesia, Pobreza Antropológica, Conocimiento Social.

\begin{abstract}
This paper summarizes some of the principal perspectives to understand poverty. I take into consideration some of the initiatives of the World Economic Forum and the United Nations, as well as some of the academia and specific research centers in this field. The scope of this article is to approach poverty from the perspective of Catholic Social Doctrine to underline that this problem is more than a material issue, it is a moral and anthropologic problem. When understood in terms of a moral and anthropologic problem, poverty is translated as violence, corruption, damage to life and to people in need etc. The point of this paper is to show that anthropologic poverty always gives place to economic poverty, but not the other way around. Economic poverty does not always generate anthropologic poverty. This is because families with few economic resources can actually live on values that allow them to have a good life. In this paper I take into consideration some of the general solutions $\mathrm{cu}^{-}$ rrently given to poverty, stressing the importance of multiplying human relationships in benefit of a greater social knowledge. The conclusions of this paper explain that people in a specific society reach development not when all of them do the same thing, but when each person is able to undertake their specific task in the best possible way.
\end{abstract}

Keywords: Poverty, Catholic Social Doctrine, Anthropologic Poverty, Social Knowledge. 


\section{POBREZA MATERIAL Y ANTROPOLÓGICA: UNA APROXIMACIÓN DESDE LA DOCTRINA SOCIAL DE LA IGLESIA}

La Doctrina Social de la Iglesia es una enseñanza vivida por millones de personas que se ocupa de problemas actuales. Esta teoría social trata

"de tales cosas que, incorporándose a la Tradición, se hacen antiguas, ofreciendo así ocasiones y material para enriquecimiento de la misma y de la vida de fe forma parte también la actividad fecunda de millones y millones de hombres, quienes a impulsos del magisterio social se han esforzado por inspirarse en él con miras al propio compromiso con el mundo"1.

En estas enseñanzas se recoge "la ciencia moral que se hace a la luz de la razon y de la fe acerca de la vida del hombre en sociedad"2. Estas enseñanzas no pretenden indicar cuáles son los instrumentos específicos para organizar la sociedad, que por lo general son instrumentos políticos o económicos, pero esta reflexión de hecho puede ayudar a quien tiene la misión de tutelar el bien común en la esfera política o económica.

En lo específico, esa ayuda puede delinearse como un marco conceptual dentro de una tradición que responde a lo más esencialmente humano. En este estudio tomaremos la Doctrina Social de la Iglesia como fundamento filosófico para estudiar la pobreza ya que, como afirman los recientes estudios sobre este problema,

"en la tarea práctica de identificar y medir la pobreza en una sociedad, se pueden elegir métodos muy diferentes, donde se pone de manifiesto la filosofía social que fundamenta esas elecciones. Puede ser una de las filosofías éticas o una de las aproximaciones que he discutido, o bien una combinación de ellas"3.

Los autores elegidos para nuestra reflexión tienen en común su atención a la dignidad de la persona como una solución para la pobreza. Los hemos elegido porque la Doctrina Social de la Iglesia comparte esta atención y reconoce además que muchos de los conceptos que nos permiten forjar las leyes y los contratos socioeconómicos provienen de una tradición anterior a la ciencia

1 Juan Pablo II (1991), p. 3.

2 Bellocq, A. (2012), p. 341.

3 Asselin, L. M. (2009), p. 190. 
económica o incluso a la política en sus términos actuales. El significado de "persona", "sociedad", "bien común", etc., que nos llevan a elaborar una jurisdicción específica y a establecer un estado de derecho forman parte del patrimonio común de la humanidad. Estos términos han sido atentamente custodiados por las diferentes tradiciones religiosas de los pueblos y, en el occidente, quien los ha preservado es la tradición judeo-cristiana.

La tradición religiosa que se encuentra a la base de la civilización occidental se ha confrontado a lo largo de los siglos con otras fuentes que la han enriquecido. Específicamente, con el derecho romano y la reflexión filosófica griega. La civilización occidental no se identifica con la religión cristiana, aunque tampoco se oponen ni se limitan mutuamente. La unidad entre creer y trabajar, servir a Dios y a la nación, orar y promover el desarrollo se encuentra en todo caso en la persona que es la que actúa, trabaja y se relaciona con los demás en la sociedad. Por este motivo el pensamiento social cristiano tiene en cuenta tanto la razón como la fe con una atención especial a la dignidad de la persona humana.

Los individuos actúan de acuerdo con sus convicciones y entre estas, la religión constituye un modelo de acción que los configura como seres humanos. Por tanto, los creyentes de una religión específica piensan sobre la realidad social de manera distinta que los creyentes de las demás religiones. Y esto puede arrojar algunas diferencias de comprensión de la realidad.

Por esta razón los romanos pontífices han recordado a los fieles de la Iglesia que, por una parte, el análisis de la sociedad forma parte de una serie de asuntos no contenidos en la revelación cristiana. Cada creyente puede forjar sus propias opiniones sobre cómo organizar mejor la sociedad aunque no coincidan con las opiniones de los demás.

Pero por otra parte, hay algunas perspectivas sociales que alcanzan una mayor sintonía con la tradición teológica de la Iglesia. La visión social más adecuada con la revelación cristiana es aquella que tiene en consideración la vida humana entendida en su sentido pleno, total. Pablo VI expresaba esta idea con estas palabras: "el desarrollo no se reduce al simple crecimiento económico. Para ser auténtico, debe ser integral, es decir, promover a todos los hombres y a todo el hombre"

4 Pablo VI (1967), n. 14. 
La pobreza es una realidad social que interesa a la Doctrina Social de la Iglesia, ya que significa condiciones de vida específicas para quienes carecen de bienestar material y de una serie de elementos necesarios para vivir una vida digna. Pero, al ser un asunto social, caben diferentes visiones que reclaman numerosos esfuerzos de la esfera política, económica y del pensamiento social de los pueblos. Al tratarse de un problema complejo, intervienen diferentes perspectivas: sociológicas, estadísticas, económicas, etc. Nuestra perspectiva específica es la de la Doctrina Social de la Iglesia y de quienes comparten esa centralidad de la persona humana. Para esto-lo subrayamos de nuevo-hemos llamado en causa numerosas fuentes que, no teniendo el mismo grado de profundidad científica, añaden en todo caso un cierto valor a esta perspectiva.

Una vez indicado el marco de referencia, conviene hacer una definición del problema.

"La pobreza consiste en cualquier forma de inequidad, que es una fuente de exclusión social, en la distribución de las condiciones de vida esenciales para la dignidad humana. Estas condiciones de vida corresponden a las capacidades de los individuos, hogares y comunidades para alcanzar sus necesidades básicas en las siguientes dimensiones: salario, educación, salud, nutrición y alimentos, salud y agua limpia, trabajo y empleo, hogar y ambiente vital, acceso a medios de producción, acceso al mercado y por último participación comunitaria y paz social" 5 .

La literatura actual, y también las enseñanzas de la Iglesia ${ }^{6}$, hablan de inequidad y no de desigualdad para subrayar la existencia una desigualdad injusta. De manera natural los seres humanos son desiguales, puesto que tienen talentos y capacidades diferentes, pero la palabra inequidad se refiere a una desigualdad que no es fruto de las condiciones naturales del hombre, sino que de alguna manera ha sido impuesta injustamente en su modo de vivir. A esta desigualdad injusta se refiere Asselin al definir la pobreza como un problema multidimensional.

\subsection{La respuesta de la esfera política.}

En nuestros días vemos con facilidad sociedades pobres. Se trata de situaciones existenciales de personas concretas que tienen a su disposición po-

5 Asselin, L. M. (2009), p. 3.

6 Cfr. Francisco I (2015b). 
cos medios materiales y pocas oportunidades de desarrollo económico, político, cultural y a veces incluso espiritual. A lo largo de los años la pobreza ha ido disminuyendo gradualmente, ya que el mundo presenta un notable crecimiento económico. Pero es innegable que la multiplicación de la riqueza no se da de la misma manera entre todos los ciudadanos.

Las Naciones se han propuesto eliminar la pobreza, fijando unos objetivos de desarrollo del milenio del 2000 al 2015 para alcanzar el desarrollo de los pueblos menos favorecidos. A estos Objetivos del Desarrollo del Milenio (Millenium Development Goals) se añadieron algunos objetivos nuevos que se referían a políticas públicas que interesaban también a las naciones más desarrolladas. A este conjunto de objetivos se le llama Objetivos del Desarrollo Sostenible (Sustainable Development Goals) y fueron diseñados para ser aplicados del 2015 al 2030.

Muchas de las políticas públicas puestas en marcha por los Objetivos de Desarrollo del Milenio del 2000 al 2015 subrayaban los problemas del desarrollo en las naciones pobres. Y por tanto el esfuerzo económico de las naciones estaba enfocado a la resolución de estas faltas: educación, servicios médicos y de higiene, atención a la desnutrición infantil y dignidad de la mujer, pobreza urbana e inseguridad, etc.

Al fijar los Objetivos de Desarrollo Sostenible, las naciones más desarrolladas se comprometieron a invertir cien mil millones de dólares entre el 2015 y el 2030. Es razonable destinar recursos fiscales para el desarrollo, pero promover el desarrollo en una nación extranjera no es siempre justificable. Los gobiernos de las naciones más desarrolladas se vieron cuestionados por el beneficio local que habían arrojado los objetivos de desarrollo del milenio entre el 2000 y el 2015 y tuvieron que aceptar que estos objetivos se referían a las naciones menos desarrolladas.

La Agenda Global de las Naciones Unidas por tanto tomó un giro para incluir algunas políticas públicas que pudieran beneficiar también a las naciones más ricas del 2015 al 2030. Por esta razón los objetivos de desarrollo sostenible son más numerosos que los del milenio, e incluyen políticas públicas que se refieren a la construcción y financiación de viviendas, ayudas económicas para favorecer el crecimiento demográfico, intercambio de información y de inversión financiera, etc. En definitiva, la Agenda Global de las Naciones Unidas indica desde hace años una serie de políticas públicas para disminuir la pobreza. 
"La agenda 2030 para el desarrollo sostenible, que incluye 17 objetivos y 169 metas, presenta una vision ambiciosa del desarrollo sostenible e integra sus dimensiones economica, social y ambiental. Esta nueva agenda es la expresion de los deseos, aspiraciones y prioridades de la comunidad internacional para los proximos 15 anos. La Agenda 2030 es una agenda transformadora, que pone a la igualdad y dignidad de las personas en el centro y llama a cambiar nuestro estilo de desarrollo, respetando el medio ambiente"

En el mensaje que el Papa Francisco dirigió a la Asamblea General de las Naciones Unidas, reconociendo el esfuerzo de las naciones, subrayaba al mismo tiempo la importancia de centrar la atención en la dignidad de la persona humana,

"la medida y el indicador más simple y adecuado del cumplimiento de la nueva agenda para el desarrollo será el acceso efectivo, práctico e inmediato, para todos, a los bienes materiales y espirituales indispensables: vivienda propia, trabajo digno y debidamente remunerado, alimentación adecuada y agua potable; libertad religiosa, y más en general libertad de espíritu y educación. Al mismo tiempo, estos pilares del desarrollo humano integral tienen un fundamento común, que es el derecho a la vida y, más en general, lo que podríamos llamar el derecho a la existencia de la misma naturaleza humana"

La carta de Papa Francisco sobre el cuidado de la casa común, llamada "Laudato Si", que tiene como tema central la justicia social y la pobreza, con una atenta consideración del problema ecológico, subraya la perspectiva de la doctrina social de la Iglesia. Con ese documento se busca "integrar la justicia en las discusiones sobre el ambiente, para escuchar tanto el clamor de la tierra como el clamor de los pobres" 9 . En el pensamiento del Papa la pobreza material está unida a una pobreza antropológica traducida en términos de exclusión social y contaminación ambiental.

\subsection{Las propuestas en la esfera económica}

Algunas de las instituciones que tienen cierta relevancia en la esfera económica colaboran seriamente en la reducción de la pobreza. Por ejemplo el

7 Naciones Unidas (2018), p. 7.

8 Francisco I (2015b).

9 Francisco I (2015), n. 48. 
Foro Económico Mundial observa con preocupación que la exclusión social se traduce en vidas humanas.

"Alrededor de 29,000 niños de menos de cinco años mueren cada día -21 al minuto- por causas superables; 2.500 millones de personas no tienen acceso a un sistema de salud adecuado; más de 1.600 millones de personas carecen de electricidad o de formas modernas de energía; alrededor del $12 \%$ de la población sufre de hambre crónica. Mientras una tercera parte del planeta vive con menos de $\$ 2$ al día, las 85 personas más ricas del mundo poseen más riqueza que la mitad más pobre del total de población mundial” ${ }^{10}$.

El World Food Program (WFP) de las Naciones Unidas opera con fondos provenientes de las 500 empresas más importantes del mundo, de acuerdo con la clasificación de Forbes. El director del WFP, David Beasley propuso a los directores generales de estas multinacionales que demostraran su interés por la sociedad, pagando al menos un día del hambre en el mundo. El WFP provee de alimento a 120 millones de personas que, si no reciben una alimentación básica inmediata, comprometerían su subsistencia. Alcanzar a estas personas que sufren de un agudo abandono social es muy caro, puesto que se encuentran en lugares muy inaccesibles y violentos, de ahí que el presupuesto diario del World Food Program alcance los noventa millones de dólares dia$\operatorname{rios}^{11}$.

El Fondo Monetario Internacional y el Banco Mundial cooperan también con las Naciones concediéndoles préstamos condicionales, de manera que quienes establecen las políticas públicas generen una mayor inclusión social. La financiación de infraestructuras como plantas generadoras de energía eléctrica o de carreteras y puertos de hecho debería facilitar el comercio y el desarrollo social.

Algunas de estas organizaciones tienen como principal objetivo permitir a las naciones invertir en infraestructuras de las que carecen. En términos de desarrollo social, cuando se superan elevados costos fijos, entonces el costo variable disminuye notablemente. Por ejemplo, una vez que se ha realizado una inversión considerable para perforar un pozo de agua y se ha construido la tubería suficiente para que el agua potable llegue a los hogares, entonces obtener agua es fácil para el individuo: basta abrir la llave del agua.

10 World Economic Forum (2015), p. 5.

11 www.wfp.org. 
En cambio, cuando no se ha invertido en esos elevados costos fijos y no existen tuberías o hay pocos pozos-es decir, cuando no se han construido infraestructuras que lleguen a los hogares-obtener agua para el individuo es muy difícil: tiene que ir al pozo, hacer fila con las personas del pueblo, transportar el agua todos los días a la casa, etc.

Las instituciones que financian estos proyectos cuentan con que algunas naciones no tienen un estado de derecho suficiente para garantizar que los fondos se utilicen de manera adecuada. Por tanto la regulación de estos fondos públicos en ocasiones se hace de acuerdo a los intereses de quienes prestan los recursos y no tanto de acuerdo con los intereses de quienes los reciben y administran. La doctrina social de la Iglesia ha denunciado esta forma de opresión social, ya que a veces se trata de

“campañas sistemáticas contra la natalidad, que, sobre la base de una concepción deformada del problema demográfico y en un clima de absoluta falta de respeto por la libertad de decisión de las personas interesadas, las someten frecuentemente a intolerables presiones... para plegarlas a esta forma nueva de opresión"12.

Además, en muchas ocasiones los recursos no son suficientes para provocar el desarrollo social y los más críticos de estas instituciones aseguran que son justamente los préstamos a las naciones menos desarrolladas lo que las mantiene en esa situación de pobreza. Moyo además afirma que estas instituciones se benefician de esa pobreza para asegurar su propia existencia.

"Sencillamente existe una presión por conceder préstamos. El banco mundial emplea 10,000 personas, el fondo monetario internacional más de 2,500; se pueden añadir otros 5,000 personas de las otras agencias de la ONU; más los empleados de por lo menos 25,000 ONG's registradas, organizaciones privadas de caridad y un ejército de agencias de ayuda gubernamentales: tomadas todas juntas emplean 500,000 personas, lo que equivale a la población de Swazilandia. Algunas veces conceden préstamos, otras veces dan donativos, pero todas están en el negocio de la ayuda social"13.

En todo caso, la esfera económica con su capacidad de medición y análisis otorga un importante enfoque al problema de la pobreza. El Banco Mun-

12 Juan Pablo II (1991), n. 39.

13 Moyo, D. (2009), p. 54. 
dial ha establecido que quienes ganan menos de dos dólares al día viven en condiciones de pobreza extrema. Esta medida económica nos permite observar que la población en pobreza extrema ha ido disminuyendo con el pasar del tiempo.

La disminución de la pobreza se debe sobre todo a las acciones de gobiernos como el de China y el de India que han permitido a más de trescientos millones de personas ganar más de dos dólares diarios en poco menos de una década. En China, "en los 20 años que siguieron a 1981, la proporción de la población viviendo en pobreza extrema cayó del 53\% al 8\%"14. El desarrollo económico en términos globales no sólo es innegable, sino positivo. No obstante, muchos indicadores muestran una multiplicación del bienestar que no siempre genera riqueza antropológica.

"En concreto, la medición de la pobreza multidimensional toma en consideración todas las dimensiones del bienestar que pueden ser relevantes (incluyendo atributos no materiales, como el estado de salud y la participación política). En cambio, un índice de pobreza material limita su atención a los fallos funcionales que se refieren a las condiciones de vida material"15.

\subsection{Algunos estudios académicos sobre la pobreza}

La pobreza es fruto de la combinación de una serie de elementos que han sido cuidadosamente estudiados por la academia. El Centro de Desarrollo Internacional de la Universidad de Harvard ha demostrado que existen algunos objetivos que son considerados necesarios para el desarrollo de las naciones y que, a pesar de haberlos conseguido, no han producido el desarrollo esperado $^{16}$. Entre estos se cuentan por ejemplo la urbanización, la educación, la tecnología, la disminución de la demografía, etc.

El nivel de educación en el mundo, medido por horas de escolaridad, ha aumentado, pero este aumento no se ha traducido en un desarrollo esperado. Lo mismo, el nivel de urbanización de los países ha aumentado, pero el crecimiento de las ciudades y la inversión en proyectos no agrícolas no ha traído el desarrollo social que se anhelaba.

Los estudios sobre la pobreza y la desigualdad nos permiten comprender que la complejidad de este problema está en que es un dilema circular. Los

14 Ravallion, M. \& Shaoua, C. (2007), p. 2.

15 Bossert, W. \& D'Ambrosio, C. (2019), p. 16.

16 Rodrik, D. (2007), p. 23. 
pueblos menos desarrollados son pobres porque no tienen educación y porque son menos urbanos que los pueblos más ricos. Pero un pueblo es pobre porque no tiene educación y porque no tiene educación es pobre. De la misma manera un pueblo es pobre porque es muy rural y pero porque es muy rural es pobre $^{17}$.

Si se toma en cuenta el problema desde la perspectiva del intercambio comercial, los países pobres son pobres porque fabrican pocas cosas y todas las cosas que fabrican las producen también los países ricos. En cambio los países ricos son ricos porque producen muchas cosas y también producen cosas que no se producen en ningún otro lugar ${ }^{18}$. En definitiva, gracias a sus estudios sobre la pobreza, la academia nos ayuda a comprender que la pobreza es un dilema circular: no hay relojeros porque no hay relojes, pero no hay relojes porque no hay relojeros.

La pobreza en estos estudios es una paradoja; por una parte las personas que se encuentran en la base de la pirámide son una gran oportunidad para el desarrollo. Por otra, a pesar de ser una fuente de oportunidad - ya que las personas más pobres de la sociedad pagan siempre más caro por algunos servicios como la atención médica, servicios financieros, etc.- los pobres no han conseguido desarrollarse.

Enriquecer la base de la pirámide sería impactar de manera positiva todo el ecosistema económico, pero esto no se ha conseguido y parece ser muy importante ya que

“entre el 2010 y el 2025, la población infantil de la África sub-sahariana aumentará en 130 millones. Esta región será también la parte del mundo con el mayor número de niños por debajo de 18 años a partir del $2030^{19}$. Se estima que la población de África se cuadruplicará para el $2100^{20}$ y los estudios de Kayizzi-Mugerwa sugieren que el camino para afrontar adecuadamente la pobreza en África es un desarrollo que incluya a todos" 21 .

Numerosos estudios buscan además establecer correlaciones entre los diferentes elementos y regiones del planeta. Por ejemplo, una de las regiones

17 Cfr. Hausmann, R. \& Hidalgo, C. (2013), p. 44.

18 Hausmann, R. \& Klinger, B. (2007), p. 3.

19 UNICEF (2014).

20 UN (2015).

21 Heshmati, A.; Rashidghalam, M. y Nilsson, P. (2019), p. 38. 
menos desarrolladas de la India, llamada Bihar, cuenta con 99 millones de habitantes. La superficie de Bihar es de casi 94 mil kilómetros cuadrados. La paradoja de la pobreza en Bihar está en que su territorio es muy fértil, y parece sencillo conseguir una buena producción agrícola en esa zona. No obstante se trata de una zona muy pobre de la India ${ }^{22}$.

$\mathrm{Al}$ tomar en cuenta otros elementos del desarrollo se sabe que la fuente de la pobreza en Bihar es la poca urbanización de esa región de la tierra. La capital de Bihar, la ciudad de Patna, tiene poco más de un millón y medio de habitantes, sólo el uno por ciento de la población vive en una gran ciudad. La correlación entre la poca urbanización y la pobreza es un hecho comprobado. El punto fundamental que conviene subrayar es que en Bihar hay poca urbanización porque las personas no se han ido a trabajar a las ciudades, dada la gran fertilidad de la tierra. Esto significa que, paradójicamente, Bihar es tan pobre porque su tierra es muy fértil.

La paradoja de la pobreza tiene, por tanto, su origen en decisiones humanas. A su nivel más básico el hombre trabaja cuando la naturaleza no le otorga todo lo que necesita para subsistir. Cuando la naturaleza otorga todos los elementos básicos de la supervivencia al mínimo esfuerzo, es más difícil el desarrollo humano.

Los estudios sobre la pobreza subrayan que se trata de un problema que excede la escasez de los recursos económicos y además que los instrumentos que desarrollan la sociedad son políticos y económicos. Pero no lo hacen de igual manera, ya que los instrumentos económicos han alcanzado una fuerza mucho mayor que los políticos por cuanto se refiere al desarrollo de los pueblos. No obstante, la economía no es una ciencia exacta, sino una ciencia social, lo que significa que en cada proceso económico conviene tener presente la intervención de la libertad humana.

\subsection{La perspectiva de la doctrina social de la Iglesia}

La pobreza es un problema múltiple que requiere una comprensión global del desarrollo social. Al mismo tiempo, tiene consecuencias antropológicas para cada individuo, como por ejemplo el esfuerzo del vivir cotidiano, que depende del nivel de desarrollo medido en términos económicos.

22 Kaplan, S. D. (2013), p. 86. 
La complejidad de los sistemas de desarrollo da lugar a zonas de pobreza también dentro de las naciones y de las ciudades desarrolladas. Más allá de regiones de pobreza marcadas, como es el caso del continente africano, estos fenómenos están presentes de un modo u otro en todas las naciones y no ha sido posible hasta el momento erradicarlos.

Quienes consideran la pobreza y la desigualdad a la luz de la tradición judeo-cristiana, elaboran una serie de distinciones.

1) El bien humano no está medido en términos de riqueza y, por tanto, la persona que se comporta de modo justo y lleva una vida buena no necesariamente recibe bienes económicos por su actitud hacia los demás. El objetivo último de la comunidad humana no es la riqueza, sino el bien común. No obstante, de hecho el bien común exige un mínimo de bienestar junto con un respeto por la propiedad privada y por la destinación universal de los bienes ${ }^{23}$. Pero la pobreza es un hecho humano indiscutible que no nos ha sido posible superar totalmente. Desde 1960 hemos gastado más de 4.6 trillones de dólares para superar la pobreza, y no sólo muchos países siguen siendo pobres, sino que hay 20 naciones que son más pobres hoy de lo que eran en $1960^{24}$.

La consecuencia de esto es que hay personas que libremente eligen vivir una vida desprendida de los bienes materiales, con una aspiración a una pobreza subjetiva independientemente de la cantidad de bienes que se posean. Esto es lo que la tradición de la Iglesia llama la virtud de la pobreza. Esta virtud es importante en nuestros días, ya que renunciar a la ostentación de los propios bienes contribuye a la paz social, así como vivir la sobriedad personal crea una cultura de la cercanía con los demás.

2) En cambio existe una pobreza subjetiva que es un mal objetivo, y es necesario superarla. Cabe aquí una segunda distinción, ya que la pobreza no es sólo una realidad material. La pobreza es un problema que implica modos de actuar, de expresarse y de pensar con consecuencias antropológicas y morales. Existe una pobreza traducida en términos de violencia, drogadicción y alcoholismo que son problemas objetivos y se encuentran en las actitudes de algunos individuos que viven de esa manera ${ }^{25}$. Esos problemas objetivos traen como consecuencia una falta de trabajo y de desarrollo económico. Por esto es posible afirmar que la pobreza antropológica da lugar a una pobreza material,

23 Cfr. Juan Pablo II (1991), n. 6.

24 Christensen, C. M.; Ojomo, E. y Dillon, K. (2019), pp. 13-14.

25 Cfr. Juan Pablo II (1991), n. 57. 
pero en cambio al revés no es siempre así, la pobreza material no necesariamente genera pobreza antropológica.

Con esto quiero afirmar que existen muchas familias de escasos recursos económicos que viven, piensan y se expresan dentro de una serie de valores y de bienes culturales que les permiten una vida buena. En cambio, cuando en una familia se acumula la pobreza moral o antropológica, por ejemplo desprecio de la mujer y de los niños, falta de higiene y sentido trascendente de la vida, entonces terminará por generarse también pobreza económica.

3) Pensar la pobreza como un conjunto de elementos humanos nos permite establecer la tercera distinción, que sirve como marco de referencia para nuestro estudio sobre la pobreza. Y esta distinción se refiere a la existencia de una pobreza material objetiva de la que no es posible o es muy difícil librarse. Esta pobreza es desproporcionada, ya que significa un despojo de todo bien material, puede ser fruto de una pobreza antropológica traducida en términos de corrupción, pero en todo caso exige una solución por parte de la sociedad. En caso de emergencias humanitarias, los pobres no pueden esperar, la sociedad tiene la responsabilidad de ayudarlos.

$\mathrm{Si}$ en cambio surge esta pobreza material objetiva de manera constante, puede ser que la corrupción del poder público o de la esfera económica sea la pobreza antropológica que denuncia la Doctrina Social de la Iglesia.

"El desarrollo de todas las personas lleva a la preocupación por superar la pobreza, establecer un comercio internacional justo y ser sensibles a las limitaciones de la ley y de sus aplicaciones en algunos países, y a una actitud firme para luchar contra la corrupción"26.

La frase más citada de Papa Francisco expresa una desproporción de intereses que pone el bienestar por encima de la persona de manera gráfica. "No puede ser que no sea noticia que muere de frío un anciano en situación de calle y que sí lo sea una caída de dos puntos en la bolsa. Eso es exclusión”27.

En resumen, el pensamiento social cristiano permite establecer algunas distinciones: la pobreza puede entenderse como una virtud, existe una pobreza subjetiva existencial—espiritual, cultural, etc.— que genera pobreza material y finalmente, la pobreza material objetiva debe ser resuelta de inmediato si es urgente, o bien, si no es urgente debe ser afrontada en una serie de dimensio-

26 Melé, D. (2015), p. 132.

27 Francisco I (2013), n. 53. 
nes que no son sólo materiales, ya que la pobreza no es un hecho económico solamente sino una realidad antropológica. Una vez establecidos estos matices podemos considerar cuál es el camino por el que la perspectiva de la Doctrina Social de la Iglesia nos lleva a pensar la pobreza en términos humanos.

\section{UN CAMINO PARA SOLUCIONAR LA POBREZA}

La Doctrina Social de la Iglesia ilumina los problemas sociales a la luz de la fe, y esto sucede en términos personales. Papa Francisco enseña que cuando una familia encuentra que sus hijos están llenos de problemas, pueden decidir que uno de los cónyuges trabaje medio tiempo para atenderlos mejor. Si esta familia decide dar ese paso, ciertamente no será una familia más rica, porque uno de los dos ganará menos, pero será una familia mejor, puesto que los hijos estarán mejor atendidos y tendrán más tiempo de uno de sus padres. Si esto es razonable para una familia, cuando en la sociedad en general vemos tantas personas con problemas serios, de alcoholismo, de violencia, de drogadicción, de adicciones a los videojuegos, etc., parecería razonable igualmente que algunas personas obtuviesen menos recursos económicos para generar riqueza antropológica allí donde no la haya.

El Papa Benedicto XVI hacía alusión a esto cuando afirmaba que "la «ciudad del hombre» no se promueve sólo con relaciones de derechos y deberes sino, antes y más aún, con relaciones de gratuidad, de misericordia y de comunión" 28 .

Es en este sentido que la doctrina social de la Iglesia recuerda a los líderes de la esfera política y económica de la sociedad que tienen una llamada específica para desarrollar la sociedad. "En los designios de Dios, cada hombre está llamado a promover su propio progreso, porque la vida de todo hombre es una vocación dada por Dios para una misión concreta"29. Y por tanto podríamos afirmar que parte de la tarea del empresario es la de cultivar la dimensión espiritual de sus trabajadores y que uno de los mayores actos de caridad que puede vivir un empresario es generar empleos.

"La actividad empresarial, que es una noble vocación orientada a producir riqueza y a mejorar el mundo para todos, puede ser una manera muy fecunda de promover la región donde instala sus emprendimien-

28 Benedicto XVI (2007), n. 6.

29 Pablo VI, (1967), p. 15. 
tos, sobre todo si entiende que la creación de puestos de trabajo es parte ineludible de su servicio al bien común" ${ }^{30}$.

Por dimensión espiritual no se entiende una dimensión necesariamente religiosa, no se trata de convertir la empresa en un centro de evangelización, sino de permitir a los trabajadores una apertura a la trascendencia, la consideración de la vida y la muerte, el saber que el trabajo humano no es nunca simplemente un acto material, sino que cada trabajador deja en aquello que hace parte de su humanidad, una huella de su propio ser.

De aquí se desprende que en la base de la comprensión del desarrollo se encuentra también el sentido de nuestro obrar social. Y cabrían tres perspectivas diferentes, fruto de una más o menos acertada comprensión de la trascendencia del hombre, por cuanto se refiere a la sociedad.

\subsection{Providencialismo y resignación}

Algunas religiones empujarían al hombre a una cierta indiferencia ante los problemas sociales. Sea porque parecería demasiado complejo el ayudar a los demás, sea porque finalmente para quien cree, la pobreza es resultado de una elección de la divinidad y no tendrá en definitiva remedio.

Esta llamada a la resignación ante los problemas sociales no es coherente con la fe cristiana. Desde hace siglos la fe de la Iglesia enseña que "nada en el mundo ocurre sin causa; la disposición natural de las cosas no es irracional, sino que está ordenada a un fin determinado (v. 7: El hombre nace para trabajar, el pájaro para volar-escribe Tomás de Aquino). El hecho de que las cosas naturales existen en vistas de un fin es el argumento más fuerte para demostrar que el mundo es gobernado por la providencia divina" ${ }^{31}$. Como consecuencia, cada individuo ha de desarrollar sus talentos de la mejor manera posible.

\subsection{Materialismo}

Una segunda perspectiva piensa que la religión no tiene relación alguna con lo social. Sus exponentes plantean que las creencias religiosas de los pueblos en realidad son un hecho privado que no tiene relación con la esfera pú-

30 Francisco I (2015), n. 129.

31 Elders, L. (2008), p. 67. 
blica, política o económica que sea. En este sentido, cada persona puede creer lo que decide creer, mientras que cumpla con las obligaciones de lo legal y tenga un sentido profesional en su trabajo.

Son ya muchos los estudios de carácter psicológico y psiquiátrico que demuestran la importancia de la persona del director de personas. Aquellos que por su profesión u oficio tienen la tarea de formar otras personas saben que las relaciones entre los hombres son lo que permiten la cohesión y el desarrollo social ${ }^{32}$.

Aristóteles pensaba que estas relaciones humanas están regidas por la política. Y observaba que la política puede entenderse de dos maneras principalmente. Por una parte el arte de la política era la tarea de los diferentes partidos que podían dialogar y confrontar sus opiniones para el mejor funcionamiento de la polis. Por otra parte, la política debería buscar el bien común y ese papel de la política no era un arte sino lo más esencial de la tarea política. Ese bien común no era otra cosa sque el bien del alma proyectado a la realidad social. Por tanto para Aristóteles la política era el arte de lo posible y pertenecía a la metafísica. Al estudio de lo que está más allá de lo observable y de lo medible por los sentidos.

En esa tradición aristotélica se encuentra el pensamiento social de los cristianos, donde se respeta una plena libertad de acción política y se recuerda una seria responsabilidad por el bien del alma, que no es nunca un bien aislado, sino que forma parte de un ser con los demás. "Ciertamente, el hombre puede organizar la tierra sin Dios, pero «al fin y al cabo, sin Dios no puede menos de organizarla contra el hombre. El humanismo exclusivo es un humanismo inhumano»" ${ }^{33}$.

\subsection{Un humanismo integral}

Finalmente, cabe la posibilidad de considerar una colaboración entre el obrar del Creador y las capacidades de las criaturas. En realidad la fe que ilumina las relaciones sociales permite observar con mayor profundidad al ser humano. Esta perspectiva permite comprender que todos los seres humanos tienen igual dignidad independientemente de su raza, sexo o condición social. $\mathrm{Y}$ por tanto, es posible respetar y hacer respetar, colaborar y conocer a todas las personas.

32 Cfr. Armenta, A. (2018).

33 Pablo VI (1967), n. 42. 
Pero además hay en esta perspectiva un deseo de llegar más lejos de lo inmediatamente necesario. Quien tiene la responsabilidad de una persona habrá de trabajar de acuerdo con las características de su compromiso: quien tiene una mujer o un marido, quien tiene un hijo o un padre o una madre que atender, quien adquiere una responsabilidad ante otra persona por una promesa o una convicción existencial, etc.

Además, habrá quien adquiera una responsabilidad más amplia, con un mayor número de personas o ante situaciones que exigen una particular atención. Es el caso, por ejemplo, de un gobernante, de un cirujano, y también de un empresario. Y la comprensión de la igual dignidad de las personas, de sus posibilidades de crecimiento, de la confianza que merecen como colaboradores llevará al líder de la organización a buscar dar más tiempo y talento a la organización que representa.

Algo similar podría pensarse en quienes comienzan organizaciones de carácter global, donde se trata no sólo de un grupo de colaboradores con fines comunes, generalmente económicos, sino de personas que comparten las mismas convicciones. Los creadores de movimientos políticos, de organizaciones sin fines de lucro, de movilizaciones ciudadanas pueden ser animados por intereses que van más allá de lo inmediato. La gradualidad del compromiso social está impulsada por la fe de los cristianos, se trata de un impulso transversal.

\subsection{La perspectiva cristiana ante la pobreza}

En resumen, el progreso social es camino natural de perfección para el hombre. Pero de hecho, este progreso no se realiza en todos los hombres. En algunas ocasiones no bastará con vivir como personas buenas, sino que además será necesario que haya estructuras sociales justas. El hombre

“está condicionado por la estructura social en que vive, por la educación recibida y por el ambiente. Estos elementos pueden facilitar u obstaculizar su vivir según la verdad. Las decisiones, gracias a las cuales se constituye un ambiente humano, pueden crear estructuras concretas de pecado, impidiendo la plena realización de quienes son oprimidos de diversas maneras por las mismas. Demoler tales estructuras y sustituirlas con formas más auténticas de convivencia es un cometido que exige valentía y paciencia" ${ }^{4}$.

34 Juan Pablo II (1991), n. 38. 
Y esta observación da lugar a una consideración de la pobreza dentro de esas dos posibilidades: la pobreza surge cuando los individuos no practican aquello que los hace excelentes, la vida virtuosa o el bien; en cambio, la pobreza sobre todo tiene cabida cuando no hay estructuras sociales justas. Ambas perspectivas dan lugar a una división por cuanto se refiere a las causas de la pobreza.

Muchas personas piensan que los pobres son pobres porque no trabajan suficiente. Se trata, en su opinión, de un problema moral. Y por lo tanto, en definitiva, para quienes así piensan, la pobreza es buscada por los pobres.

En cambio, hay muchas otras personas que consideran que la pobreza no tiene su fuente principal en la virtud de las personas, sino en las condiciones sociales en que existen. Parecería que personas con una gran capacidad de esfuerzo se ven limitadas por las condiciones a hacer lo posible para sobrevivir. En este segundo caso, la pobreza es una desafortunada condición existencial.

Estas perspectivas de comprensión llevan a diferentes consideraciones sociales. Quien piensa que los pobres son pobres porque lo han decidido de esa manera, no considera la pobreza como una responsabilidad suya, ya que los pobres han optado por vivir así. Tal vez no es una opción consciente, tal vez les falta información, o educación, o virtud, pero al final del camino son decisiones personales las que llevan cada persona a la pobreza. Quien por el contrario piensa que los pobres son pobres porque la estructura social los mantiene pobres, desea hacer algo más para solucionar esa situación, puesto que es una condición en la que los pobres han nacido y no pueden liberarse por si mismos.

Las estructuras sociales no se identifican con las convicciones personales. Para adquirir una convicción es necesario pensar, justamente considerar si aquello que se vive, se expresa o se piensa en una sociedad determinada es lo correcto, lo justo o lo mejor. Y hacerlo significa pensar nuevamente en el sentido del porqué se dice y se hace lo que de hecho se vive en una sociedad determinada.

Gran parte de los ciudadanos de los Estados Unidos piensan que los pobres dejarían de serlo si se empeñaran en trabajar más. En cambio, gran parte de los ciudadanos franceses consideran que la pobreza es fruto de la disfunción institucional del Estado. Tal vez no les falta razón a quienes piensan que la intervención estatal en materia de política monetaria, inflacionaria y fiscal son las causas principales de la pobreza. 
La perspectiva de la Iglesia tiene que ver un poco más con las convicciones de los hombres que con las estructuras sociales. Para la perspectiva de la Doctrina Social de la Iglesia que intentamos seguir es más importante el modo en que consideramos la pobreza, que la pobreza en sí misma. Porque de hecho, si se piensa que la pobreza es culpa de los pobres, entonces no se tiene ninguna responsabilidad ante este agudo problema social. Por el contrario, si se piensa que la pobreza es una realidad social fruto de estructuras injustas, entonces es responsabilidad de cada uno hacer algo para cambiar esta condición humana.

Conviene no olvidar que la pobreza es una pobreza antropológica, no sólo material. Y que esa pobreza antropológica puede ser resuelta por ambos elementos, la bondad de las personas y la justicia de las estructuras. Todos los individuos tenemos una cierta pobreza antropológica y es tarea de todos considerar los medios necesarios para cambiar, para hacer algo. Cuando se ignora esa tarea de la conversión social es cuando se cae en el individualismo, en la indiferencia.

Esto es lo que recientemente denuncia el Papa Francisco cuando afirma que en nuestros días

"se considera al ser humano en sí mismo como un bien de consumo, que se puede usar y luego tirar. Hemos dado inicio a la cultura del «descarte» que, además, se promueve. Ya no se trata simplemente del fenómeno de la explotación y de la opresión, sino de algo nuevo: con la exclusión queda afectada en su misma raíz la pertenencia a la sociedad en la que se vive, pues ya no se está en ella abajo, en la periferia, o sin poder, sino que se está fuera. Los excluidos no son «explotados» sino desechos, «sobrantes»" 35 .

\section{LA POBREZA Y SUS SOLUCIONES}

Si hasta ahora hemos hablado de perspectivas de comprensión, conviene señalar igualmente que no basta con comprender los problemas del mundo, sino que es necesario poner los medios para solucionarlos. La vida buena, la moral que enseña la Doctrina Social de la Iglesia no es una comprensión más profunda de las realidades sociales, sino una guía para transformar la vida cotidiana en una vida con sentido trascendente, que vaya más allá.

35 Francisco I (2013), n. 53. 
Sería oportuno considerar las razones por las cuales no se ha resuelto la pobreza si la tecnología ha avanzado tanto en las últimas décadas. Se comprende que el desarrollo de los pueblos tiene un método bastante estudiado por la ciencia económica y política. Es ante la dificultad que tienen algunas regiones de la tierra para desarrollarse que podríamos considerar aquello que observaba Karl Popper:

"no hay un número límite de observaciones de cisnes blancos, para probar la teoría de que todos los cisnes son blancos. Pero basta observar un sólo cisne negro para desaprobar esta teoría" ${ }^{\prime 36}$

La mayor parte de las regiones de la tierra han comenzado ese camino hacia el desarrollo, pero no todas lo han conseguido. Esto tal vez podría significar, parafraseando a Popper, que la observación del desarrollo en un elevado número de naciones, no garantiza que sea el único camino a seguir para el desarrollo, puesto que de hecho hay regiones de la tierra donde esto no ha funcionado.

El hecho de haber encontrado un cisne negro entre los muchos blancos que se han observado tampoco significa que en realidad los cisnes blancos no lo fueran. Por esta razón Hausmann afirma que sería equivocado juzgar erróneas las teorías del desarrollo simplemente porque algunas zonas de la tierra no han alcanzado el progreso. "La diferencia enorme de ingresos entre los países pobres y los ricos son una expresión de las gran diferencia de conocimientos acumulados que han ido amasando las naciones" ${ }^{37}$.

La Doctrina Social de la Iglesia por su parte, no condena sistemas económicos en términos generales, sino que pretende comprender el sentido que se les da a esos sistemas. Ante la pregunta si el capitalismo es el mejor sistema económico para el florecimiento humano, Juan Pablo II afirmaba,

“Si por «capitalismo» se entiende un sistema económico que reconoce el papel fundamental y positivo de la empresa, del mercado, de la propiedad privada y de la consiguiente responsabilidad para con los medios de producción, de la libre creatividad humana en el sector de la economía, la respuesta ciertamente es positiva, aunque quizá sería más apropiado hablar de «economía de empresa», «economía de mercado», o simplemente de «economía libre». Pero si por «capitalismo» se entiende un sistema en el cual la libertad, en el ámbito económico, no está

36 Popper, K. (1954), p. 101.

37 Hausmann,R. \& Hidalgo, C. (2013), p. 7. 
encuadrada en un sólido contexto jurídico que la ponga al servicio de la libertad humana integral y la considere como una particular dimensión de la misma, cuyo centro es ético y religioso, entonces la respuesta es absolutamente negativa" ${ }^{38}$.

Por estos mismos motivos los pastores de la Iglesia no califican de inmoral un sistema económico, ni se pronuncian contra políticas públicas complejas sin antes definir el sentido de su preocupación. Los documentos escritos por los pontífices podrían equipararse a una queja pública hecha por alguien cargado con un profundo sentido de humanidad. En palabras más sencillas, sería como un padre que al notar el dolor de su hijo alza su voz, pero lógicamente ese padre lleva a su hijo a un médico especialista, no intenta curarlo personalmente. Dejando atrás nuestro ejemplo, la Doctrina Social de la Iglesia no indica soluciones concretas, simplemente tiene la misión de recordar que no todo está bien, que hemos de pensar cómo superar la exclusión social, sin dar soluciones específicas a problemas opinables.

\subsection{Las teorías de desarrollo social}

Algunas teorías del desarrollo social han superado la idea de que para desarrollar alguna región bastaría con la acumulación de bienes económicos. Robert Solow, por ejemplo, recuerda que la combinación de capital (entendido como los elementos necesarios para la producción), los trabajadores y un cierto conocimiento social permiten alcanzar el desarrollo ${ }^{39}$. Hausmann e Hidalgo-tomando como base la reflexión de Solow—proponen una novedosa teoría del desarrollo social centrada en la persona y en su capacidad de interactuar con los demás.

"La cantidad de conocimiento contenida en una sociedad, en todo caso, no depende principalmente de cuánto conocimiento tenga cada individuo. En cambio depende de la diversidad del conocimiento entre muchos individuos y en su habilidad de combinar este conocimiento, y hacer uso de él, a través de redes complejas de interacción" ${ }^{* 0}$.

En primer lugar esta teoría subraya con acierto que el capital no es una acumulación monetaria, sino la creación de instrumentos que faciliten la producción. La infraestructura que permite los transportes, la maquinaria que fa-

38 Juan Pablo II, (1991) Centesimus Annus, 42)

39 Solow, R. (1956), p. 67.

40 Hausmann,R. \& Hidalgo, C. (2013), p. 15. 
cilita el trabajo en el campo, etc. Al mismo tiempo, recuerda que un trabajador no produce el doble porque pueda manejar dos máquinas. Existe siempre un factor humano importante en el desarrollo que no se puede ignorar.

Probablemente el factor más complejo esta teoría es el del conocimiento. $\mathrm{Y}$ este se decanta en tres formas de comprender el conocimiento. En primer lugar el conocimiento está en los instrumentos. Un teléfono celular o un tractor contienen conocimientos de ingeniería, de análisis de materiales, de diseño gráfico y de cálculo estructural. Además para fabricar esos instrumentos fue necesario contar con grandes conocimientos de física, ingeniería eléctrica y mecánica, etc. Pero usar un teléfono celular o un tractor no requiere tanta ciencia, basta mucho menos para echarlos a andar. Por lo tanto los instrumentos acumulan en sí mismos mucho conocimiento, al menos como se entiende el conocimiento bajo esta teoría del desarrollo.

La solución de la pobreza no está simplemente en llevar instrumentos allí donde no los hay. Podría asumirse que no todas las personas saben utilizar los instrumentos, pero además que estos instrumentos necesitan de otros instrumentos para funcionar. Utilizar un tractor supone la existencia de gasolineras, talleres de reparación de tractores, empresas que vendan ruedas y refacciones, caminos para transportar los tractores, etc. Si no se cuenta con una larga serie de servicios que permitan la funcionalidad del tractor, éste no podrá generar riqueza.

Podría por tanto suponerse que los instrumentos exigen un cierto conocimiento para ser operativos. Bastaría por tanto con alertar a los nuevos usuarios de instrumentos cómo deben manejar los instrumentos y cuáles son los elementos necesarios para su buen uso. Hausmann recuerda que el conocimiento se encuentra en estas instrucciones de uso de los instrumentos, es lo que llama conocimiento como códigos.

De hecho en nuestros días la mayor parte, si no la totalidad, de los códigos de uso de los instrumentos de que dispone comúnmente la sociedad se encuentran disponibles gracias al internet. No obstante, a pesar de la cantidad de códigos disponibles no se ha superado la pobreza de manera general.

La teoría del desarrollo social de Hausmann, basada en el conocimiento humano, no supone que la pobreza se abate sencillamente trasladando herramientas y códigos a las regiones del planeta donde no los hay. La razón es que cuando una persona tiene una necesidad no actúa utilizando personalmente las herramientas a disposición, aunque sea posible aprender los códigos para usar esas herramientas. El ejemplo con el que ilustra esta realidad es la de una 
persona con dolor de muelas, que en lugar de comprar el instrumental dental y aprender por internet cómo extraer una muela, acude al dentista ${ }^{41}$.

Para estos economistas el conocimiento está por tanto también acumulado en los expertos que dominan las herramientas y los códigos. La sociedad, cuando ha ido acumulando experiencia suficiente, consigue el desarrollo gracias a la interacción entre los diferentes expertos. Tristemente los países subdesarrollados están hundidos en la pobreza no sólo porque tengan carencias de herramientas y de códigos, sino sobre todo porque aquellos que consiguen ser expertos en algún campo del saber humano, generalmente consiguen su experiencia fuera de las regiones pobres, y no vuelven a ellas.

Es fácil mover herramientas y códigos a las zonas donde no hay desarrollo, pero es muy difícil mover los expertos a las regiones en vías de desarrollo, porque esa decisión es fruto de la libertad humana. La conclusión de esta teoría es clara,

"no tiene sentido pasar nuestra vida aprendiendo cómo hacerlo todo. Puesto que es difícil de transferir, el conocimiento tácito es lo que limita el proceso de crecimiento y desarrollo. Al final, las diferencias en prosperidad están relacionadas con la cantidad de conocimiento tácito que tienen las sociedades"42.

\subsection{El especial campo de acción de los creyentes}

Para Hausmann un experto no es alguien que sabe conceptualmente muchas cosas, sino uno capaz de poner en práctica aquella experiencia acumulada por la destreza de los instrumentos y códigos que desarrollan la sociedad. Ser experto no es poseer mucho conocimiento sobre la realidad, sino actuar en ella con soltura y dominarla.

Daniel Goleman (2008) afirma que el ser humano opera la mayor parte del tiempo con una inteligencia operacional automática, mientras que sólo en algunos momentos del día es necesario poner especial atención en alguna actividad específica. Una sociedad experta es la que funciona de manera habitual con esa inteligencia automática, puesto que sus miembros poseen la virtud del desarrollo de manera adquirida. Aristóteles y Tomás de Aquino asegurarían que una sociedad virtuosa es la que lleva a cabo cotidianamente su

41 Cfr. Hausmann,R. \& Hidalgo, C. (2013), p. 15.

42 Hausmann,R. \& Hidalgo, C. (2013), p. 16. 
obrar con hábitos operativos buenos y el bien común político es la adquisición de las virtudes que nos permiten vivir en una buena comunidad humana.

Joshua Greene, por su parte, reconoce que el cerebro humano elabora su conocimiento como una cámara fotográfica que por lo general capta en modalidad panorámica la realidad. No obstante, en ciertos momentos, es posible adaptar el uso del cerebro como se hace en modo manual en una cámara para fijar la atención sobre algún asunto específico y registrarlo de manera especial. Para este psicólogo cuando el cerebro opera de manera habitual de acuerdo con aquello que es mejor para la sociedad, se consigue una sociedad inteligente, habituada al desarrollo.

"Como los ajustes automáticos de una cámara, las emociones producen un comportamiento que por lo general se adapta, sin la necesidad de un pensamiento consciente sobre lo que hay que hacer. Y también como los ajustes automáticos de una cámara, el diseño de las respuestas emocionales-el modo en que estas ubican los inputs del ambiente como modos de comportarse-incorporan las enseñanzas de experiencias pasadas"43.

Si estas teorías-que tienen su origen en el funcionamiento del cerebro humano y en el comportamiento social observable-son correctas, entonces podríamos afirmar que un experto tenista no sabe jugar al tenis. $\mathrm{O}$ bien, que es un experto en el tenis, pero que sería muy difícil que explicara exactamente cuáles son los músculos que debe mover cuando observa la pelota acercarse a él a una velocidad determinada. También sería muy difícil explicar la magnitud de fuerza con que debe pegar a la pelota y la inclinación de la raqueta ante una situación de ángulo determinado, etc. El conocimiento de los expertos no es comprensión, sino la capacidad adquirida de obrar habitualmente de manera excelente.

Por ese mismo motivo, no sería posible aprender a jugar un deporte, escuchando las indicaciones teóricas de un experto. Para practicar de manera excelente un deporte, el camino adecuado es entrenarse con un experto, convivir con él, moverse con él. Pero los expertos no se encuentran fácilmente al alcance de aquellos que no lo son, su motivación es la de acompañar en su profesión a otros expertos del mismo campo.

El Papa Francisco recoge esta profunda idea con palabras sencillas, cuando afirma que al dar limosna no sólo basta con tirar la moneda, sino que

43 Greene, J. (2013), pp. 134-135. 
hemos de tocar la mano y mirar a los ojos. "Necesitamos saber encontrarnos. Necesitamos edificar, crear, construir, una cultura del encuentro" ${ }^{44}$.

Establecer un diálogo entre aquellos que son expertos y quienes no lo son es tarea fundamental de los creyentes, sean expertos o no. La interacción social es lo que desarrolla el mundo y para conseguirla no basta con una simple lógica económica o política, es siempre necesaria una perspectiva plenamente humana, que es propia de la Doctrina Social de la Iglesia y de quienes contemplan la posibilidad de la acción concreta ante Dios en este mundo.

\subsection{Interacción humana y saber hacer social}

Los fieles de la Iglesia saben que han de estar dispuestos a ser expertos en humanidad y a colaborar con todos en la construcción de un mundo más humano. Esta interacción social se facilita cuando cada persona se ocupa de su propia tarea en servicio de los demás. No se trata de que todas las personas realicen la misma labor, sino de que cada persona aprenda a hacer algo distinto $^{45}$.

Cuando Vasco de Quiroga evangelizó los pueblos indígenas de una región de Michoacán en México, enseñó a los habitantes de pequeñas aldeas una profesión específica. En su idea, era importante que cada pueblo hiciera algo diferente de los demás pueblos, para facilitar el comercio, el intercambio de bienes y conocimientos y en definitiva el desarrollo. De esta manera algunos pueblos como Paracho son conocidos por sus guitarras, otros por sus sombreros o su producción ganadera, etc. Cada pueblo desarrollo una profesión diferente que les permitía llevar a cabo un comercio con las demás poblaciones indígenas de aquella zona.

En las sociedades más rudimentarias, cada persona sabe hacer muchas cosas: cazar animales, pescar, elaborar trineos, construir casas, hacer fuego, etc. En cambio en las sociedades más avanzadas, cada persona sabe hacer menos cosas: escribir libros, enseñar alguna materia, trabajar en la construcción, etc. La diferencia está en que los habitantes de las sociedades más desarrolladas saben hacer menos cosas porque en las sociedades ricas hay más personas que saben hacer cosas de manera diferente. El conocimiento que se encuentra en el "ser expertos" de las personas no significa que cada persona sabe hacer más cosas, sino que cada persona sabe hacer lo que hace de manera diferente.

44 Francisco I (2013).

45 Cfr. Hausmann,R. \& Hidalgo, C. (2013), p. 15. 
Tanto en las sociedades ricas como en las sociedades más pobres se vende fruta. Pero en las sociedades pobres una sola persona planta, cosecha, riega y recoge la fruta, después la lleva al mercado y la vende. En todo el proceso de venta de fruta en una sociedad rudimentaria hay una persona que actúa. Y consigue hacer lo que puede. En cambio en una sociedad desarrollada la venta de fruta implica un grupo de expertos que siembran, otro diferente que coloca la fruta en envases para su venta, otro grupo de personas que la transportan a puntos de venta especializados. En la sociedad desarrollada vender fruta significa la interacción de un gran número de expertos que no saben hacer lo que los demás hacen, pero que saben hacer diferente. Como consecuencia, en la sociedad desarrollada la eficiencia de la venta de fruta se multiplica por la acumulación de un gran "saber hacer social".

Cuando se piensa que una sociedad puede desarrollarse a fuerza de aumentar las personas que hacen las mismas cosas, como por ejemplo en una planta de fabricación textil donde cada empleado hace lo mismo que los demás, no se da el desarrollo. El desarrollo se alcanza cuando cada individuo produce un bien diferente del bien que producen los demás, y especialmente cuando el bien individual suma al bien colectivo la experiencia de lo excelente.

De esta manera, el mayor desarrollo personal significa al mismo tiempo un mayor desarrollo de la comunidad humana. Una de las imágenes más claras para conseguirlo es la de una orquesta, ante la excelencia de cada uno de los músicos aumenta la excelencia de la orquesta y el renombre de la orquesta es fuente de seguridad y satisfacción para cada uno de los músicos. Cabrían también otros ejemplos, la universidad y sus profesores, un hospital y sus médicos, etc. En realidad se trata de aquella definición profunda del bien común que daba hace siglos Tomás de Aquino y que puede resumirse como "el orden que luce en la comunidad como resultado de la instauracion en la multitud, de la vida virtuosa, y la preeminencia de la vida contemplativa" 46 .

La Doctrina Social de la Iglesia invita a cada individuo a desarrollar la sociedad como una llamada divina. Pero este desarrollo no se realiza sólo por una acción instrumental, sino que se alcanza cuando cada persona se empeña en su desarrollo personal al servicio del bien común. No se trata de crear sociedades cada vez más eficientes, sino de fomentar espacios de interacción y convivencia con los expertos, esta es la perspectiva más esencialmente humana

46 Raffo Magnasco, B. (1949), p. 2026. 
del desarrollo. La pobreza económica-fruto de la pobreza moral—tiene su punto final en el encuentro sincero entre los hombres.

\section{BIBLIOGRAFIA}

Armenta, Alejandro (2018), Hablar al hombre del hombre: El director como persona, LID Editorial \& IPADE Publishing, Ciudad de México.

Asselin, Louis-Marie (2009), Analysis of Multidimensional Poverty: Theory and Case Studies. Economic Studies in Inequality, Social Exclusion and Well-Being, Springer, Ottawa.

Bellocq, Arturo (2012), "Que es y que no es la Doctrina Social de la Iglesia: una propuesta”, Scripta Theologica, vol.44, pp. 337-366.

Bossert, Walter \& D’Ambrosio, Conchita (2019), “Intertemporal Material Deprivation: A Proposal and an Application to EU Countries", en Dasgupta, Indraneel \& Manipushpak, Mitra (eds.), Deprivation, Inequality and Polarization: Essays in Honour of Satya Ranjan Chakravarty, Springer, New York, pp. 15-35.

Concilio Vaticano II (1967), Gaudium et Spes, Constitución pastoral sobre la Iglesia en el mundo actual en www.vatican.va

Christensen, Clayton M.; Ojomo, Efosa; Dillon, Karen (2019), The Prosperity Paradox: How Innovation Can Lift Nations Out of Poverty, Harper Collins, New York.

Elders, Leo (2008), Conversaciones Teológicas con Santo Tomás de Aquino, Verbo Divino, Mendoza.

Francisco I (2013), Evangelii Gaudium Sobre el anuncio del Evangelio en el mundo actual.www.vatican.va

Francisco I (2015), Laudato Si. Sobre el cuidado de la casa común, www.vatican.va

Francisco I (2015b), Discurso del Santo Padre a la Organización de las Naciones Unidas. 25 septiembre 2015. Disponible en www.vatican.va

Goleman, Daniel (2008), The Secret to Success. Education Digest: Essential Readings Condensed for Quick Review, vol. 74, no 4, pp. 8-9. 
Greene, Joshua (2013), Moral Tribes: Emotion, Reason, and the Gap Between Us and Them, Penguin Press, New York.

Hausmann, Ricardo \& Hidalgo, Cesar (2013), The Atlas of Economic Complexity: Mapping Paths to Prosperity, MIT University Press, Cambridge.

Hausmann, Ricardo \& Klinger, Bailey (2007), "The Structure of the Product Space and the Evolution of Comparative Advantage", CID Working Papers 146, Center for International Development at Harvard University.

Heshmati, Almas; Rashidghalam, Masoomeh, \& Nilsson, Pia (2019), "Measurement and Analysis of Multidimensional Well-Being in Rwanda", en Nilsson, Pia y Heshmati, Almash (eds.), Efficiency, Equity and Well-Being in Selected African Countries, Economic Studies in Inequality, Social Exclusion and WellBeing, Springer Nature, Switzerland, pp. 37-68.

Juan Pablo II (1991), Centesimus Annus. En el centenario de la Rerum Novarum, www.vatican.va.

Kaplan, Seth D. (2013), Betrayed: Power, Politics and Prosperity, Palgrave McMillan, New York.

Melé, Domènec (2015), “Three Keys Concepts of Catholic Humanism for Economic Activity" en Schlag M., Melé D. (eds.), Humanism in Economics and Business: Perspectives of the Catholic Social Tradition, Issues in Business Ethics vol. 43. Springer: New York, pp. 113-136.

Moyo, Dambisa (2009), Dead Aid: Why Aid is Not Working and How There is a Better Way for Africa, Farrar, Strauss and Giroux, New York.

Naciones Unidas (2018), La Agenda 2030 y los Objetivos de Desarrollo Sostenible: una oportunidad para America Latina y el Caribe (LC/G.2681-P/Rev.3), Santiago. disponible en www.cepal.org

Pablo VI (1967), Populorum Progressio. Sobre la necesidad de promover el desarrollo de los pueblos.

Popper, Karl R. (1954), “The Problem of Induction”, en Miller David (ed.) (1985), Popper Selections 101, Princeton University Press, Princeton.

Raffo Magnasco, Benito (1949), "Bien comuún y políitica en la concepción filosofica de Santo Tomás de Aquino”, Actas del Primer Congreso Nacional de Filosofia, Mendoza, Argentina, vol. 3, pp. 2022-2032.

Ravallion Martin y Shahoua, Chen (2007), "China's (uneven) Progress Against Poverty", Fournal of Development Economics, vol. 82, pp. 1-42. 
Rodrik, Dani (2007), "Industrial Development: Some Stylized Facts and Policy directions", en United Nations, Department of Economic and Social Affairs. Industrial Development for the 21st Century: Sustainable Development Perspectives, United Nations, New York.

Solow, Robert (1956), "A Contribution to the Theory of Economic Growth", The Quarterly Fournal of Economics, vol. 70, n 1, pp. 65-94.

World Economic Forum (2015), Towards a New Global Consciousness, Global Agenda, REF 140115. 
